\title{
Modification of the Generalized Born Model Suitable for Macromolecules
}

\author{
Alexey Onufriev, Donald Bashford, and David A. Case* \\ Department of Molecular Biology, The Scripps Research Institute, La Jolla, California 92037 \\ Received: November 16, 1999; In Final Form: January 27, 2000
}

\begin{abstract}
The analytic generalized Born approximation is an efficient electrostatic model that describes molecules in solution. Here it is modified to permit a more accurate description of large macromolecules, while its established performance on small compounds is nearly unaffected. The modified model is also adapted to describe molecules with an interior dielectric constant not equal to unity. The model is tested by computations of $\mathrm{p} K$ shifts for a number of titratable residues in lysozyme, myoglobin, and bacteriorhodopsin. In general, except for some deeply buried residues of bacteriorhodopsin, the results show reasonable agreement with both experimental data and calculations based on numerical solution of the Poisson-Boltzmann equation. A very close agreement between the two models is obtained in an application to the prediction of the $\mathrm{p} K$ shifts associated with conformational change. The calculations based on this version of the generalized Born approximation are much faster than finite difference solutions of the Poisson-Boltzmann equation, which makes the present method useful for a variety of other applications where computational time is a critical factor. The model may also be integrated into molecular dynamics programs to replace explicit solvent simulations which are particularly time-consuming for large molecules.
\end{abstract}

\section{Introduction}

Over the past ten years classical electrostatic models based upon numerical solution of the Poisson-Boltzmann (PB) equation have been successfully applied to compute various properties of macromolecules. ${ }^{1-9}$ However, since solving a system of partial differential equations is a computationally costly procedure, the method may become quite time-consuming, especially if applied to a large set of independent conformations of a macromolecule, or if it is incorporated into molecular dynamics programs.

Recently, several fast analytic versions of the so-called generalized Born (GB) approximation have been proposed as an alternative to the computationally intensive PoissonBoltzmann approach. ${ }^{10-15}$ For small molecules, the method has been shown to reproduce solvation energies and individual charge-charge interactions very well when compared to solutions of the Poisson-Boltzmann equation. ${ }^{11,12,14-16}$ However, in similar comparisons on larger molecules, the agreement has not been as close, the discrepancy generally being more pronounced for molecules having large interior regions. ${ }^{15,16}$ There, the generalized Born approximation tends to overestimate the solvation energy of deeply buried atoms and to underestimate the interaction between them, as compared to numerical solution of the Poisson-Boltzmann (PB) equation. ${ }^{15,16}$ This appears to be a general property of this type of GB approximation, independent of a particular parametrization. The resulting error in the calculation of solvation energies is often acceptable, since the atoms contributing the most to the solvent polarization energy are the ones on the surface, and they are the most accurately treated. Also, for an overall neutral molecule, the individual errors in like-charge interactions largely cancel those coming from opposite-charge ones. ${ }^{15}$ However, when an accurate estimate of the interactions between individual atoms becomes important, such as in calculations of $\mathrm{p} K$ shifts, the

* Corresponding author. E-mail: case@scripps.edu. method is likely to show poorer performance. ${ }^{12}$ For example, the GB approximation was recently applied ${ }^{17}$ to the estimation of $\mathrm{p} K$ shifts of the active-site aspartic dyad in HIV-1 protease. It was found that the set of atomic radii that worked well for small molecules did not produce accurate estimates for the two residues considered, and had to be adjusted. Proteins represent a significant challenge to the GB approximation as they generally contain biologically important functional groups which may lie both on the surface and deep in the molecular interior. Hence, a simple adjustment of atomic radii that may improve the method's performance for buried residues is likely to be inadequate for the well-exposed ones, and vice versa. The theory clearly needs to be improved to correctly describe interior regions of large molecules while preserving its remarkable accuracy already established for small compounds.

In this work we develop an analytic GB theory which is suitable for proteins. We begin by re-deriving the GB approximation for the general case of a molecular interior dielectric that is not necessarily equal to unity. We then formulate a simple criterion which any GB theory must satisfy in order to correctly describe interior atoms. Application of the criterion to the pairwise GB method leads us to introduce a single new parameter into the model, to account for the nonzero size of solvent molecules. We optimize its value by comparing the charge-charge interactions in myoglobin calculated by the GB model with those obtained by numerical solution of the $\mathrm{PB}$ equation. We then study the performance of the modified GB model by computing $\mathrm{p} K_{1 / 2}$ values for ionizable groups in lysozyme, myoglobin, and bacteriorhodopsin. The predicted values agree reasonably well with both experiment and calculations based upon solution of the PB equation. The agreement between the two models becomes even better when the GB approach is used to evaluate the difference in titration behavior associated with conformational change. We also show that the corrections have little effect on GB accuracy for small molecules, allowing one to use the same set of parameters for all 
compounds, regardless of size. Our current implementation of the GB method is significantly faster than its finite-difference PB counterpart when applied to titration calculations on medium-sized proteins such as lysozyme.

\section{Theory}

The continuum electrostatic model, ${ }^{1}$ which is the starting point for both the GB and PB models, subdivides the entire space into two regions separated by a dielectric boundary: the solute, characterized by a low dielectric value, and the solvent, which has a high dielectric value. The electrostatic potential can then be calculated directly, without any other approximations, by solving the Poisson-Boltzmann equation numerically to a desired degree of accuracy. Alternative methods, such as the modified generalized Born model considered below, are based on various further approximations that allow one to obtain an analytical form of the potential or the electrostatic component of the solvation free energy for a complex molecule. The accuracy of the GB approximation can be assessed by comparing its predictions to the corresponding PB results.

2.1. GB Model with Variable Interior Dielectric. We represent each atom in the molecule as a sphere of radius $\rho_{i}$ with a charge $q_{i}$ at its center; the interior of the atom is assumed to be filled uniformly with dielectric material of low dielectric constant $\epsilon_{\mathrm{p}}$. The molecule is surrounded by a solvent of a high dielectric value $\epsilon_{\mathrm{w}}$. The electrostatic component of the free energy is the work $W$ of creating a given charge distribution. Its calculation is made nontrivial by the presence of surface polarization that develops at the dielectric boundary between the solute and the solvent if $\epsilon_{\mathrm{p}} \neq \epsilon_{\mathrm{w}}$. We single out the part of $W$ that is due to the polarization charges by considering a process in which the molecule is transfered from a uniform medium of dielectric value $\epsilon_{\mathrm{p}}$ into the solvent with dielectric constant $\epsilon_{\mathrm{w}}$ :

$$
W=W^{0}+\Delta W
$$

where $W^{0}$ is the energetic cost of creating the charge distribution in a uniform dielectric $\epsilon_{\mathrm{p}}$ and $\Delta W$ is the energy cost of the transfer, i.e., the solvation energy. The evaluation of $W^{0}$ is straightforward since no surface polarization is present; it is simply the Coulomb charge-charge interaction:

$$
W^{0}=\frac{1}{2} \sum_{i \neq j} \frac{q_{i} q_{j}}{\epsilon_{\mathrm{p}} r_{i j}}
$$

An efficient way to compute $\Delta W$ is given by the generalized Born model which is conventionally applied ${ }^{10,14-16}$ to the case $\epsilon_{\mathrm{p}}=1$. The theory is, however, not specific ${ }^{19}$ to this particular value of $\epsilon_{\mathrm{p}}$, and one can easily modify it to describe the more general case of $\epsilon_{\mathrm{p}} \neq 1$ :

$$
\Delta W=-\frac{1}{2}\left(\frac{1}{\epsilon_{\mathrm{p}}}-\frac{1}{\epsilon_{\mathrm{w}}}\right) \sum_{i, j} \frac{q_{i} q_{j}}{j_{i j}^{\mathrm{gb}}\left(r_{i j}\right)}
$$

where $f_{i j}^{\mathrm{gb}}$ is a certain smooth function which is assumed to depend only upon atomic radii $\rho_{i}$ and interatomic distances $r_{i j}$. A detailed analysis of the approximations on which the model is based is presented in ref 19 . Here we motivate eq 3 by considering the exact analytical form of $\Delta W$ for a pair of atoms $i$ and $j$ in the limiting cases of $r_{i j} \rightarrow \infty$ and $r_{i j} \rightarrow 0$, and requiring that $f_{i j}^{\mathrm{gb}}$ interpolate between the two extremes.

When the atoms are far apart, the work done on transferring the pair from the medium of dielectric constant $\epsilon_{\mathrm{p}}$ into the one of $\epsilon_{\mathrm{w}}$ must be

$$
\lim _{r_{i j} \rightarrow \infty} \Delta W=-\frac{1}{2}\left(\frac{1}{\epsilon_{\mathrm{p}}}-\frac{1}{\epsilon_{\mathrm{w}}}\right)\left(\frac{q_{i}^{2}}{\rho_{i}}+\frac{q_{j}^{2}}{\rho_{j}}\right)-\left(\frac{1}{\epsilon_{\mathrm{p}}}-\frac{1}{\epsilon_{\mathrm{w}}}\right) \frac{q_{i} q_{j}}{r_{i j}}
$$

The first term is the Born solvation energy of transferring the two independent spherical ions between the two media, and the second term corresponds to the difference in the interaction energy of two point charges in the two media. In the other extreme $r_{i j} \rightarrow 0$, the two spheres merge into one, and if we also assume that $\rho_{i} \approx \rho_{j}$, the system may be approximated by a sphere of radius $\left(\rho_{i} \rho_{j}\right)^{1 / 2}$ with total charge $\left(q_{i}+q_{j}\right)$. Therefore, $\Delta W$ in this case is just the Born solvation energy of transferring the sphere between the two media:

$$
\lim _{r_{i j} \rightarrow 0} \Delta W=-\frac{1}{2}\left(\frac{1}{\epsilon_{\mathrm{p}}}-\frac{1}{\epsilon_{\mathrm{w}}}\right)\left(q_{i}+q_{j}\right)^{2}\left(\rho_{i} \rho_{j}\right)^{-1 / 2}
$$

The function $f_{i j}^{\mathrm{gb}}$ in eq 3 should hence be such that $f_{i i}^{\mathrm{gb}}=\rho_{i}$. As $r_{i j} \rightarrow \infty$, we require $f_{i j}^{\mathrm{gb}} \rightarrow r_{i j}$, and as $r_{i j} \rightarrow 0, f_{i j}^{\mathrm{gb}} \rightarrow\left(\rho_{i} \rho_{j}\right)^{1 / 2}$. The fact that these requirements on $f_{i j}^{\mathrm{gb}}$ depend only on the geometry of the system and not on the dielectric constant allows us to use exactly the same $f_{i j}^{\mathrm{gb}}$ here as in the standard formulation of GB with $\epsilon_{\mathrm{p}}=1$, which is conventionally considered in literature. This is important, since $f_{i j}^{\mathrm{gb}}$ and the associated set of atomic parameters have already been optimized by others. The standard $^{11}$ form of $f_{i j}^{\mathrm{gb}}\left(r_{i j}\right)$ is particularly simple:

$$
f_{i j}^{\mathrm{gb}}=\left[r_{i j}^{2}+R_{i} R_{j} \exp \left(-r_{i j}^{2} / 4 R_{i} R_{j}\right)\right]^{1 / 2}
$$

where $R_{i} \geq \rho_{i}$ is the so-called effective Born radius which replaces $\rho_{i}$ and accounts for the fact that atoms $i$ and $j$ may be surrounded by neighbors that displace the solvent and therefore decrease the polarization energy. A method to estimate $R_{i}$, which is the crux of our modification to the GB theory, is presented in section 2.2. Larger effective radii result in smaller contributions to $\Delta W$; their values reflect the extent of burial and depend only on the mutual positions, radii, and types of the surrounding atoms. In the limiting cases described above, and with other neighbors far away, we require that $R_{i} \rightarrow \rho_{i}$, and one can check that eqs 4 and 5 are satisfied with $f_{i j}^{\mathrm{gb}}$ from eq 6.

Given $\Delta W$ from eq 3 , the total electrostatic work $W$ of creating the given charge distribution in the solvent is

$$
W=-\frac{1}{2}\left(\frac{1}{\epsilon_{\mathrm{p}}}-\frac{1}{\epsilon_{\mathrm{w}}}\right) \sum_{i, j} \frac{q_{i} q_{j}}{f_{i j}^{\mathrm{gb}}\left(r_{i j}\right)}+\frac{1}{2} \sum_{i \neq j} \frac{1}{\epsilon_{\mathrm{p}}} \frac{q_{i} q_{j}}{r_{i j}}
$$

2.2. Evaluation of Effective Radii. One way to estimate the effective Born radius $R_{i}$ is to consider the change $\Delta W_{i}$ in the self-energy of atom $i$ upon solvation. According to eqs 3 and 6:

$$
\Delta W_{i}=-\frac{1}{2}\left(\frac{1}{\epsilon_{\mathrm{p}}}-\frac{1}{\epsilon_{\mathrm{w}}}\right) \frac{q_{i}^{2}}{R_{i}}
$$

On the other hand, this quantity can also be calculated directly on the basis of classical electrostatics. ${ }^{18,20}$ The work done on creating a given charge distribution in an arbitrary dielectric environment is

$$
W=\frac{1}{8 \pi} \int_{R^{3}} \frac{[\vec{D}(\vec{r})]^{2}}{\epsilon(\vec{r})} \mathrm{d}^{3} \vec{r}
$$


where $\vec{D}(\vec{r})$ is the dielectric displacement vector, and $\epsilon(\vec{r})$ is the position-dependent dielectric constant. Therefore, the work $\Delta W_{i}$ of transferring the atom $i$ from a medium of uniform dielectric constant, $\epsilon_{\mathrm{p}}$, to the two-dielectric solute/solvent system is

$$
\begin{aligned}
& \Delta W_{i}=\frac{1}{8 \pi \epsilon_{\mathrm{w}}} \int_{\text {solvent }}\left[\vec{D}_{i}(\vec{r})\right]^{2} \mathrm{~d}^{3} \vec{r}+ \\
& \frac{1}{8 \pi \epsilon_{\mathrm{p}}} \int_{\text {solute }}\left[\vec{D}_{i}(\vec{r})\right]^{2} \mathrm{~d}^{3} \vec{r}-\frac{1}{8 \pi \epsilon_{\mathrm{p}}} \int_{\text {solvent }}\left[\vec{D}_{i}^{0}(\vec{r})\right]^{2} \mathrm{~d}^{3} \vec{r}- \\
& \quad \frac{1}{8 \pi \epsilon_{\mathrm{p}}} \int_{\text {solute }}\left[\vec{D}_{i}^{0}(\vec{r})\right]^{2} \mathrm{~d}^{3} \vec{r}
\end{aligned}
$$

where $\vec{D}_{i}(\vec{r})$ is the total dielectric displacement due to charge $i$, and

$$
\vec{D}_{i}^{0}(\vec{r}) \equiv \frac{q_{i}}{r^{3}}
$$

is the Coulomb field created by point charge $q_{i}$ in the uniform dielectric environment. For convenience we have placed the origin of the coordinate system at the center of atom $i$. So far, eq 10 is an exact result. We now make the approximation:

$$
\vec{D}_{i}(\vec{r}) \approx \vec{D}_{i}^{0}(\vec{r}) \equiv \frac{q_{i}}{r^{3}} \vec{r}
$$

The validity of the approximation above is considered in detail in refs 19 and 20 . On substituting $\vec{D}_{i}(\vec{r})$ from eq 12 into eq 10 , the integrals over the solute volume cancel, while the solvent volume integrals combine:

$$
\begin{aligned}
\Delta W_{i}=\frac{1}{8 \pi}\left(\frac{1}{\epsilon_{\mathrm{w}}}-\frac{1}{\epsilon_{\mathrm{p}}}\right) \int_{\text {solvent }}\left[\vec{D}_{i}^{0}(\vec{r})\right]^{2} \mathrm{~d}^{3} \vec{r}= \\
\quad\left(\frac{1}{\epsilon_{\mathrm{w}}}-\frac{1}{\epsilon_{\mathrm{p}}}\right) \frac{q_{i}^{2}}{8 \pi} \int_{\text {solvent }} \frac{1}{r^{4}} \mathrm{~d}^{3} \vec{r}=-\frac{1}{2}\left(\frac{1}{\epsilon_{\mathrm{p}}}-\frac{1}{\epsilon_{\mathrm{w}}}\right) \frac{q_{i}^{2}}{R_{i}}
\end{aligned}
$$

where $R_{i}$ is an effective radius defined by

$$
R_{i}^{-1}=\frac{1}{4 \pi} \int_{\text {solvent }} \frac{1}{r^{4}} \mathrm{~d}^{3} \vec{r}
$$

The integration domain in the above equation can be changed to the solute volume, which is computationally more convenient. Note that

$$
\begin{aligned}
\frac{1}{\rho_{i}} & =\frac{1}{4 \pi} \int_{R^{3}} \frac{1}{r^{4}} \theta\left(|\vec{r}|-\rho_{i}\right) \mathrm{d}^{3} \vec{r} \\
& =\frac{1}{4 \pi} \int_{\text {solute }} \frac{1}{r^{4}} \theta\left(|\vec{r}|-\rho_{i}\right) \mathrm{d}^{3} \vec{r}+\frac{1}{4 \pi} \int_{\text {solvent }} \frac{1}{r^{4}} \mathrm{~d}^{3} \vec{r}
\end{aligned}
$$

where $\theta\left(|\vec{r}|-\rho_{i}\right)$ is the step function. Therefore, the effective Born radius $R_{i}$ is also given by

$$
R_{i}^{-1}=\rho_{i}^{-1}-\frac{1}{4 \pi} \int_{\text {solute }} \theta\left(|\vec{r}|-\rho_{i}\right) \frac{1}{r^{4}} \mathrm{~d}^{3} \vec{r}
$$

As expected, $R_{i}=\rho_{i}$ if the solute consists of only one atom $i$.

2.2.1. Integration Domain. Up until this point, the derivation has not depended on the exact definition of the solute or solvent volume. To proceed with the evaluation of the effective Born radius, we need to specify the boundaries of the integration domain in eq 16. In the PB calculations, it is common to define the low dielectric solute as the region bounded by the molecular surface. ${ }^{21}$ With this definition, most small crevices between the

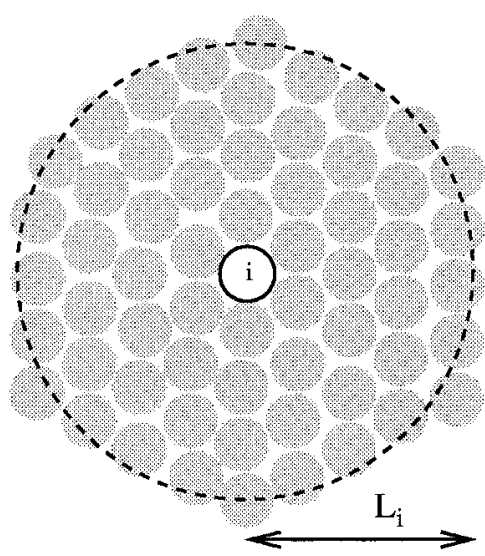

Figure 1. Schematic of a macromolecule, with the circles representing the atomic VDW spheres. $L_{i}$ is the shortest distance from the atom $i$ to the molecular surface. For a roughly spherical molecule with the atom $i$ in its center, the generalized Born radius should be $R_{i} \approx L_{i}$.

van der Waals (VDW) spheres of protein atoms fall in the solute region because a solvent-sized probe cannot fit into them. This implies that one could implement eq 16 by numerical integration over the volume bounded by the molecular surface. ${ }^{11,22}$ The procedure would, however, undermine the main advantages of the analytic GB model that were outlined in the Introduction, as it would involve finding the molecular surface and performing a costly numerical integration. To circumvent the problem, the analytic GB method performs the integral in eq 16 over the VDW spheres of atoms. This implies a definition of the solute volume in terms of a set of spheres, rather than the molecular surface, and allows one to obtain ${ }^{14}$ an analytical form for $R_{i}$. The approach is known to work well in the case of small molecules, but for large compounds such as proteins it has been shown ${ }^{15,16}$ to overestimate the solvation energy of deeply buried atoms as well as to underestimate the interaction between them, as compared to the PB calculations that use the molecular surface-based definition of solute. One plausible reason for this behavior is that, in the sphere-based GB method, only the inside of each sphere has a low dielectric value and all crevices in between are filled with high dielectric solvent. For small molecules, this distinction is unimportant, but for large macromolecules that have considerable interior regions from which solvent is completely expelled, it results in the effective dielectric constant of the molecular interior being too high. In other words, the effective Born radii are underestimated for deeply buried atoms. The theory apparently needs to be modified to correctly describe, within the accuracy of the continuum approximation, the electrostatics of the interior regions of large molecules, while retaining its good performance for small molecules.

2.2.2. Packing Correction Factor. We begin by analyzing the correct behavior of the effective Born radius as an atom becomes buried deep inside the solute, and then modify the analytic GB model to reproduce it. Consider a large macromolecule and assume that its interior is totally inaccessible to the solvent, the situation corresponding to the molecular surfacebased definition of solute volume. The effective Born radius of any buried atom $i$ must then be no smaller than the shortest distance $L_{i}$ between the atom and the molecule-surface interface. For the roughly spherical molecular surface of Figure 1 , the integral in eq 16 can be approximated analytically and we obtain $R_{i} \approx L_{i}$. For a nonspherical molecule, the total volume of the low dielectric region around atom $i$ is always larger than that corresponding to the inscribed sphere of radius $L_{i}$, and therefore the generalized Born radius of the atom must satisfy the condition 


$$
R_{i} \geq L_{i}
$$

Physically this condition implies that if $L_{i} \rightarrow \infty$ the atom does not "see" the solvent and its Born solvation energy $\Delta W_{i}$ in eq 13 must tend to zero. Any GB theory must satisfy this general criterion in order to correctly describe large, densely packed molecules.

A GB approximation does not satisfy eq 17 if the integral in eq 16 is taken only over the solute volume based on VDW spheres, instead of the molecular surface-based volume; we miss the interatomic spaces inaccessible to solvent in Figure 1, resulting in an underestimation of $R_{i}$. To correct this, we introduce a new parameter $\lambda>1$ into eq 16 :

$$
R_{i}^{-1}=\rho_{i}^{-1}-\frac{\lambda}{4 \pi} \int_{\mathrm{VDW}} \theta\left(|\vec{r}|-\rho_{i}\right) \frac{1}{r^{4}} \mathrm{~d}^{3} \vec{r}
$$

where the integral is now taken over the VDW volume of the spheres (shaded regions in Figure 1), and the $\lambda$ factor compensates for the missing volume. For an initial guess of $\lambda$, consider the hypothetical case of a very large molecule made up of identical atoms, the atom $i$ being in its center, as in Figure 1. Since even in the densest packing (without overlaps) of identical spheres, only three-fourths of the total space is occupied, choosing $\lambda \sim 4 / 3$ should approximately compensate for the missed volume, and bring eq 18 in better accord with condition eq 17. The assumption of the dense packing is, in fact, a very reasonable one for proteins. ${ }^{23,24}$ Physically, the value of the packing correction factor $\lambda>1$ accounts for nonzero size of the solvent molecule.

As for the effect of this correction on surface atoms and small molecules, note that $\mathrm{d} R_{i} / \mathrm{d} \lambda \sim R_{i}\left(R_{i} / \rho_{i}-1\right)$ for $\lambda \sim 1$, so that only large effective radii of deeply buried atoms change significantly, while the small ones of the surface atoms change only slightly, since for them $R_{i} \approx \rho_{i}$. Therefore, for our calculations we can use nearly the same set of atomic parameters as in earlier works, such as in ref 15 , while making the GB model more suitable for interior regions by optimizing the value of $\lambda$; see below.

2.2.3. Parameterization of the Model. We employ the approach of Hawkins et al. ${ }^{14}$ and approximate the effective Born radius of atom $i$ by calculating analytically the contribution of each atom $j$ to the integral in eq 18 and adding the contributions together. Since the procedure ignores overlaps among the atoms surrounding atom $i$, we follow Hawkins et al. ${ }^{15}$ and introduce empirical scaling factors $S_{j}$ that partially account for this behavior. Here we adopt the model that the $S_{j}$ 's depend only on the identity of atom $j$, with values given elsewhere. ${ }^{15}$ Following Still et al. ${ }^{11}$ we begin the calculation of effective Born radii with atomic radii reduced slightly from those used in the corresponding numerical PB calculations; the offset is $\rho_{0}=0.09$ $\AA$. We have already mentioned that setting $\lambda \sim 1.33$ is expected to have very little effect on individual charge-charge interactions in a small molecule. However, since all of the effective radii increase with $\lambda$, and since we also wish to retain the remarkable accuracy of the GB in solvation energy calculations, we shift all of the effective radii calculated via eq 19 downward by a small term $\delta=0.15 \AA$ in the end of the calculation: $R_{i} \rightarrow$ $R_{i}-\delta$. This term is purely empirical and allows us to avoid complete reparametrization of the model, such as readjustment of $S_{j}$ or $\rho_{0}$. Note that setting $\delta=0.15 \AA$ has little effect on the large radii $R_{i} \gg 1 \AA$ of buried atoms.

Introducing the sum over $i$ and $j$ and the above parameters into eq 18 , we write

$$
R_{i}=\left[\left(\rho_{i}-\rho_{0}\right)^{-1}-\lambda \sum_{j} H\left(r_{i j}, S_{j}\left(\rho_{j}-\rho_{0}\right)\right)\right]^{-1}-\delta
$$

where $H\left(r_{i j}, S_{j}\left(\rho_{j}-\rho_{0}\right)\right)$ represents the result of integration (scaled by $r^{-4}$ ) over the VDW volume of atom $j$; its analytical form is given in ref 14 and we use it here.

We model the effects of ionic strength by simple substitution

$$
\left(\frac{1}{\epsilon_{\mathrm{p}}}-\frac{1}{\epsilon_{\mathrm{w}}}\right) \rightarrow\left(\frac{1}{\epsilon_{\mathrm{p}}}-\frac{\mathrm{e}^{-\kappa f_{i j} j^{b}}}{\epsilon_{\mathrm{w}}}\right)
$$

in eq 7 , where $\kappa$ is the Debye-Hückel screening parameter. This approximation was introduced earlier ${ }^{15}$ and was shown to work reasonably well. Finally, we notice that since the free energy $W$ in eq 7 is a quadratic function of charges, we can express it in terms of the GB analogue of the electrostatic potential $\phi\left(\vec{r}_{i}\right)$ defined at the position of each atom $i$ :

$$
\phi\left(\vec{r}_{i}\right)=-\left(\frac{1}{\epsilon_{\mathrm{p}}}-\frac{\mathrm{e}^{-\kappa f_{i j}^{\mathrm{g} b}}}{\epsilon_{\mathrm{w}}}\right) \sum_{j} \frac{q_{j}}{f_{i j}^{\mathrm{gb}}\left(r_{i j}\right)}+\sum_{j \neq i} \frac{1}{\epsilon_{\mathrm{p}}} \frac{q_{j}}{r_{i j}}
$$

These potentials enter into the titration calculations described below. Unless otherwise specified, we use $\epsilon_{\mathrm{w}}=80$ and $\epsilon_{\mathrm{p}}=4$. To model physiological conditions in our test calculations, we set $\kappa^{-1}=10 \AA$ which corresponds to about a $0.1 \mathrm{M}$ solution of a monovalent salt. The present form of the GB approximation permits straightforward introduction of a cutoff, where only the atoms within a specified cutoff distance are taken into account when computing both the effective Born radius and the atomatom interactions for a given atom.

2.2.4. Titration Calculations. The approach used here to calculate the electrostatic contributions to titration in proteins has been described in detail elsewhere..$^{2,29-31}$ It is assumed that the difference in the titration behavior of an ionizable group in a protein and in a model compound can be accounted for by calculating the difference in the electrostatic work of altering the charges from the unprotonated to the protonated state in the protein and the work of making the same alteration in the model compound. In its original formulation for the PB model, this quantity is expressed through the values of the electrostatic potential at the atoms' positions. Therefore, the same formalism can be applied verbatim for the GB method once the GB potential analogue is defined via eq 21 .

The protonation fraction of each site at any particular value of the $\mathrm{pH}$ can be obtained by considering a Boltzmann-weighted sum over all possible protonation states of the protein, or in the case of a large number of sites, by a suitable approximation method. In the present work, either the reduced site method ${ }^{32}$ or a Monte Carlo method ${ }^{33}$ is used. The $\mathrm{pH}$ at which the protonation fraction of a site is 0.5 is then reported as the calculated $\mathrm{p} K$ (or $\mathrm{p} K_{1 / 2}$ ) of the site.

We have incorporated the GB method into the MEAD suite of programs, ${ }^{26,31}$ where it optionally replaces the finite difference solver for calculating electrostatic interactions. The codes and input files necessary to reproduce the calculations presented in this work will be made available for download through the Web site, http://www.scripps.edu/bashford, with the next public release of MEAD.

\section{Results and Discussion}

3.1. Optimization of $\lambda$. We begin by optimizing the value of the correction factor $\lambda$ introduced above by comparing the charge-charge interaction matrix $W_{i j}$ calculated by the GB 


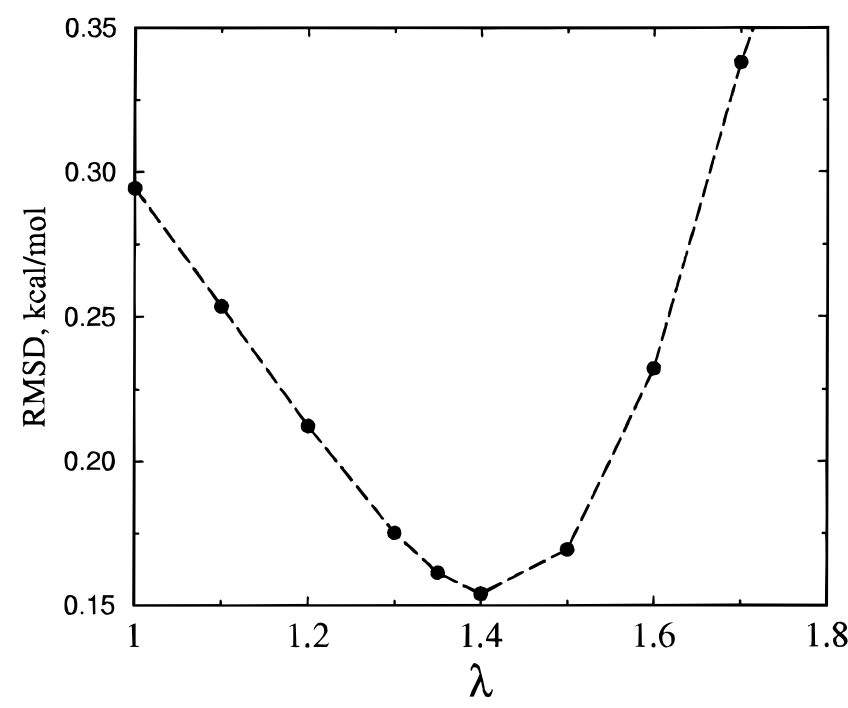

Figure 2. rmsd between charge-charge interaction energies $W_{i j}$ in myoglobin calculated within GB and PB models for different values of $\lambda$. The rmsd is computed as $\left((1 / N) \sum_{i j}\left(W_{(i j)}^{\mathrm{GB}}-W_{(i j)}^{\mathrm{PB}}\right)^{2}\right)^{1 / 2}$ over a randomly selected set of $N \approx 30000$ pairs of atoms.

approach with the one obtained by solving the PB equation using the same set of coordinates, charges, radii, and internal dielectric constant. The interaction is calculated as

$$
W_{i j}=q_{i} \phi^{j}\left(\vec{r}_{i}\right)
$$

where $\phi^{j}\left(\vec{r}_{i}\right)$ is the electrostatic potential due to atom $j$ at the position of atom $i$; for the GB model it is given by eq 21 with a fixed value of $j$.

We choose myoglobin as a test case, since it has a large interior. The methodology used for obtaining the pairwise interactions by the PB approach is identical to that of ref 15 . As in the previous work, we use the partial atomic charges from the AMBER force field ${ }^{27}$ and the standard Bondi radii. ${ }^{28}$ Atomic coordinates are taken from the $1.5 \AA$ X-ray structure. ${ }^{34}$ The interior dielectric constant $\epsilon_{\mathrm{p}}$ is set to 1.0. The GB calculations for different values of $\lambda$ are performed, and the root-mean-square deviation (rmsd) between the PB and GB values for the $W_{i j}$ are calculated (Figure 2). The two models are in the best agreement when $\lambda \approx 1.4$, which is close to our initial guess of $\lambda=1.33$ derived from simple packing considerations

3.2. Pairwise Interaction Energies. We have assessed the accuracy with which the modified GB model reproduces the PB results for charge-charge interactions in small molecules. Figure 3 illustrates the performance of the $\lambda=1.4 \mathrm{~GB}$ model relative to the $\mathrm{PB}$ calculations by plotting the charge-charge interaction energies in aspartic acid calculated using the GB theory vs those calculated using the PB model for the same pairs of atoms. The numbers predicted by the two methods are nearly identical. Similar results for the original $(\lambda=1) \mathrm{GB}$ model were reported earlier. ${ }^{15}$ Solvation energies of small molecules calculated using the modified GB theory approximate those obtained by the PB model reasonably well: for aspartic acid, glutamic acid, and a GLU-GLU dipeptide in a standard extended conformation the relative differences are 5\%, 4\%, and $2.5 \%$ respectively.

For larger compounds, we expect ${ }^{15}$ larger deviations between the GB and PB approaches, and this is indeed seen in Figure 4 (left panel); but as one would expect from Figure 2, the modified GB model (Figure 4, right panel) agrees with the PB calculations more closely than the original GB model. The distribution of points around the $x=y$ line (perfect match) is narrower, especially for larger energies, in the case $\lambda=1.4$ than for the original model corresponding to $\lambda=1.0$.

It is also instructive to compare the values of the so-called effective dielectric constant $\epsilon_{i j}$ between two atoms $i$ and $j$, computed using different models. This quantity is defined so that $W_{i j}=q_{i} q_{j} /\left(\epsilon_{i j} r_{i j}\right)$. Smaller values of $\epsilon_{i j}$ correspond to buried atoms characterized by larger charge-charge interactions. We compute this quantity using the same set of $W_{i j}$ as before, and plot the resulting $\epsilon_{i j}$ as a function of charge-charge separation (Figure 5). As expected, the original GB model with $\lambda=1.0$ significantly overestimates $\epsilon_{i j}$ (underestimates the charge-charge interactions) for buried atoms relative to the PB calculations, while the $\lambda=1.4$ theory shows considerable improvement. Note, however, that setting $\lambda=1.4$ does not necessarily bring the GB model into exact agreement with the general criterion formulated earlier, eq 17 . This value of $\lambda$ merely represents the optimum for the current model. Increasing $\lambda$ even further to $\lambda$ $=1.7$ (Figure 5, bottom left) brings the charge-charge interactions of the most deeply buried atoms (low $\epsilon_{i j}$ ) closer to the corresponding PB values, but worsens the overall agreement between the two models, as shown in Figure 2. This is most likely due to an overestimation of the effective Born radii of surface atoms; the GB model originally was parametrized to perform well only on small molecules.

3.3. $\mathbf{p} K$ Calculations. We have assessed the performance of the modified GB model by calculating titration curves for a number of individual residues in lysozyme, sperm whale carbonmonoxymyoglobin, and bacteriorhodopsin. All of these proteins have titratable groups both on the surface and in the interior regions, and in the case of bacteriorhodopsin some of the residues exhibit extremely large $\mathrm{p} K$ shifts. Theoretical $\mathrm{p} K$ calculations based on the PB model for these proteins have been reported previously, 2,30,31 and we follow the general methodology presented in those references. Our primary objective is to assess the performance of the modified GB model, mainly relative to the established $\mathrm{PB}$ approach. The results are reported in terms of a single number for each residue, the $\mathrm{p} K_{1 / 2}$, which is the midpoint of its titration curve. ${ }^{31}$

In all titration calculations we use the standard Bondi radii set, and use $\epsilon_{\mathrm{p}}=4.0, \epsilon_{\mathrm{w}}=80.0$. Unless otherwise specified we choose $\lambda=1.4, \delta=0.15$ in the GB calculations, and a probe radius of $1.4 \AA$ to compute the dielectric boundary in the PB method. Only single-conformer calculations are performed for each structure.

3.3.1. Lysozyme. A first set of calculations was made using the coordinates of the triclinic form of hen egg lysozyme as determined by neutron scattering ${ }^{35}$ (PDB Accession No. OLZ5). Since hydrogen atom positions are available in this case, we perform no further manipulation on the structure and assign atomic charges according to the standard AMBER classification scheme. In contrast to the original lysozyme $\mathrm{p} K$ calculations, which used a single-charge model of the titrating sites, ${ }^{2}$ we use a full set of partial charges for both the protonated and deprotonated forms of the sites. We calculate $\mathrm{p} K_{1 / 2}$ values for 21 titratable residues in lysozyme using the GB model, and compare the results with experimental data and PB calculations in Figure 6a. The GB method gives a good overall agreement with experiment. It correctly predicts the $\mathrm{p} K_{1 / 2}$ values for most residues and reproduces the trends in the experimentally observed $\mathrm{p} K_{1 / 2}$ shifts, such as the pronounced downward shift for ASP-48 and ASP-66 or an upward shift of TYR-53. It is also important to notice that the GB $\mathrm{p} K_{1 / 2}$ values are highly correlated with the $\mathrm{PB}$ ones for all residues, even 


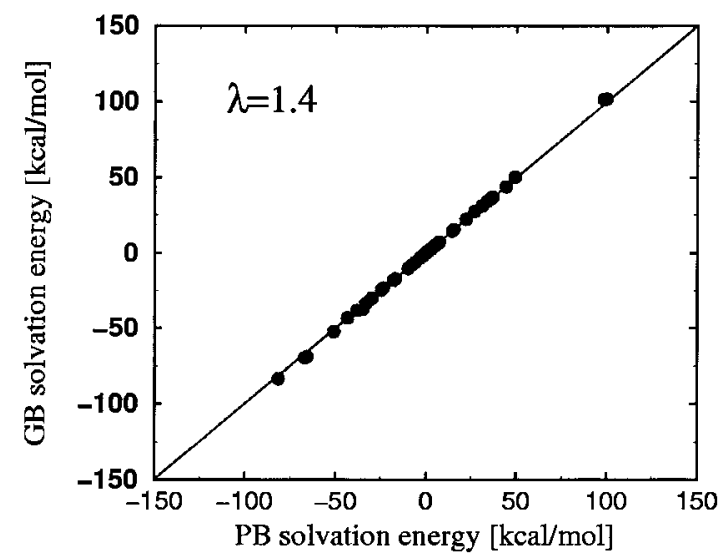

Figure 3. Comparison of the polarization part of the electrostatic charge-charge interactions $\Delta W_{i j}$ in aspartic acid for $\lambda=1.4 \mathrm{~GB}$ and PB models, computed over all pairs of atoms. The line $x=y$ represents a perfect match between the two models. The results show a very close agreement over the entire range of energies.

where the calculated results deviate from experiment. ${ }^{36}$ To better illustrate the point, we plot $\left(\mathrm{p} K_{1 / 2}-\mathrm{p} K_{\text {mod }}\right)$ ( $\mathrm{p} K$ shifts relative to the corresponding model compound) for both methods in Figure 6b. The correlation between the PB and GB models is rather good, even for residues showing $\mathrm{p} K$ shifts as large as 5. These observations strongly suggest that the GB approach presented here is comparable in accuracy to the accepted PB method in predicting the titration properties of triclinic lysozyme, while the deviations from experiment point out the limitations of the single conformer continuum electrostatic model itself. ${ }^{37-39}$ Introduction of a $12 \AA$ cutoff does not affect the predicted values of $\mathrm{p} K$ shifts significantly: the standard deviation over 21 residues between the two calculations is $0.34 \mathrm{p} K$ units, and all trends in $\mathrm{p} K$ shifts are preserved, while the speed of the computation is doubled. Although the $12 \AA$ cutoff has not introduced any significant error into these calculations, in other contexts the use of cutoffs may be problematic.

Next, we have tested the performance of the GB model for evaluating the change in titration behavior associated with conformational change. We have calculated the $\mathrm{p} K_{1 / 2}$ values for the same set of 21 residues using coordinates taken from the X-ray structure ${ }^{40}$ of the tetragonal crystal form of lysozyme (PDB Accession No. 193L). Since hydrogen atom positions are not available, we use the LEAP utility of the AMBER package to add them. The goal is to see how structural differences between the two forms of the same protein affect the calculated $\mathrm{p} K_{1 / 2}$ values. Although the backbone rmsd between the two structures, $0.71 \AA$, is small, the calculated $\left(\mathrm{p} K_{1 / 2}^{\mathrm{tetr}}-\mathrm{p} K_{1 / 2}^{\mathrm{tric}}\right)$ is quite significant for some residues, as shown in Figure 7. This sensitivity of calculated $\mathrm{p} K$ values to local conformational changes has been noted previously. ${ }^{2,30}$ As seen in Figure 7, the GB predictions follow the PB ones very closely, the correlation coefficient being 0.994 . The agreement between GB and PB theories is better for calculating the difference in $\mathrm{p} K_{1 / 2}$ between conformers than for finding the absolute values of $\mathrm{p} K_{1 / 2}$.

As has already been mentioned, the analytic GB model is considerably faster than the PB one. Although a direct comparison is somewhat arbitrary since it depends on the adjustable accuracy of the numerical computations, we find ${ }^{41}$ that a $\mathrm{p} K$ calculation on lysozyme as described above takes $\approx 8$ min by the GB method, vs $\approx 86$ min by the PB method which uses successive over-relaxation algorithm to solve the finitedifference representation of the PB equation on two levels of focusing, each having 81 lattice points in each dimension. Introduction of a $12 \AA$ cutoff into the GB model roughly doubles its speed.

3.3.2. Myoglobin. We have performed titration calculations on the sperm whale carbonmonoxymyoglobin $1.5 \AA \mathrm{X}$-ray structure $^{34}$ (PDB Accession No. 1MBC), and compared the GB and $\mathrm{PB}$ results to each other and to available experimental data. We have used the AMBER charges, and the methodology of the calculation follows the earlier work on myoglobin presented in ref 30; we treat all ARG, LYS, TYR, GLU, and ASP residues plus $\mathrm{C}$ and $\mathrm{N}$ termini as titratable groups, and we account for the $\epsilon$ and $\delta$ tautomers of all histidine residues. To make a direct comparison with available ${ }^{42}$ experimental data, we list the results for histidines in Table 1. A noteworthy feature of the experimental results is that three histidine residues (HIS-24, -64, and -82) do not titrate in the $\mathrm{pH}$ range in which the protein is stable. In the native myoglobin these residues are poorly exposed to solvent, and their protonation is coupled to unfolding of the protein, which occurs below $\mathrm{pH} 4$. The GB calculation shows the best agreement with experiment for $\lambda=1.4$, with all $\mathrm{p} K_{1 / 2}$ values being within two $\mathrm{p} K$ units from experimental values, and most within one unit, which is better than the corresponding $\mathrm{PB}$ result shown in the first column of Table 1 . Notably, the $\mathrm{PB}$ and $\mathrm{GB}$ values deviate from experiment in the same direction, as in the case of lysozyme considered above. All major trends in the $\mathrm{p} K$ shifts are correct.

For comparison, we have performed GB calculations with different values of the packing correction factor $\lambda$. The $\lambda=1.0$ model, corresponding to the original GB formulation, incorrectly predicts near-normal $\mathrm{p} K_{1 / 2}$ values for HIS-24, -64, and -82, while both experiment and the PB calculations show considerable downward shift for these residues. Such behavior of $\lambda=1.0$ model is expected since the original GB model underestimates the effect of burial. Setting $\lambda=1.7$, on the other hand, brings the $\mathrm{p} K_{1 / 2}$ of the nontitratable buried histidines closer to the PB values, but results in too low $\mathrm{p} K_{1 / 2}$ values for HIS-12, $-48,-113$, and -119 which are known experimentally to have near-normal $\mathrm{p} K_{1 / 2}$ values. Overall, the $\lambda=1.4$ model appears to be optimal for myoglobin, in agreement with our analysis in the previous section.

It is also interesting to see see how the size of a water molecule (i.e., the solvent probe radius) in the PB model affects the $\mathrm{p} K$ calculations. This parameter is absent from the original GB theory, and we expect that the $\lambda=1.0 \mathrm{~GB}$ model should be closest to the PB model with the probe radius set to zero. To this end, we compare the $\mathrm{p} K_{1 / 2}$ values obtained using the default probe radius of $1.4 \AA$ to those obtained with zero probe radius (Table 1). The zero-probe-radius $\mathrm{PB}$ model produces much smaller $\mathrm{p} K$ shifts, comparable to those predicted by the GB $\lambda=1.0$ model. This is not surprising since in the latter case both models assume that all space beyond the atomic radii is filled with solvent, including small internal crevices. When a nonzero probe radius is used in the PB model, there are pockets of free space in the protein interior that are not filled with solvent, and that therefore have a low dielectric constant. This enhances charge-charge interactions resulting in larger $\mathrm{p} K$ shifts from their model compound values. Setting $\lambda>1$ in the GB model tends to mimic, in an average sense, this enhancement of charge-charge interactions characteristic of the PB model with nonzero probe radius.

3.3.3. Bacteriorhodopsin. Accurate prediction of the titration properties of biologically important residues in bacteriorhodopsin has been a challenge to the theoretical community for almost a decade, and different models have been proposed..$^{31,43,44}$ The 

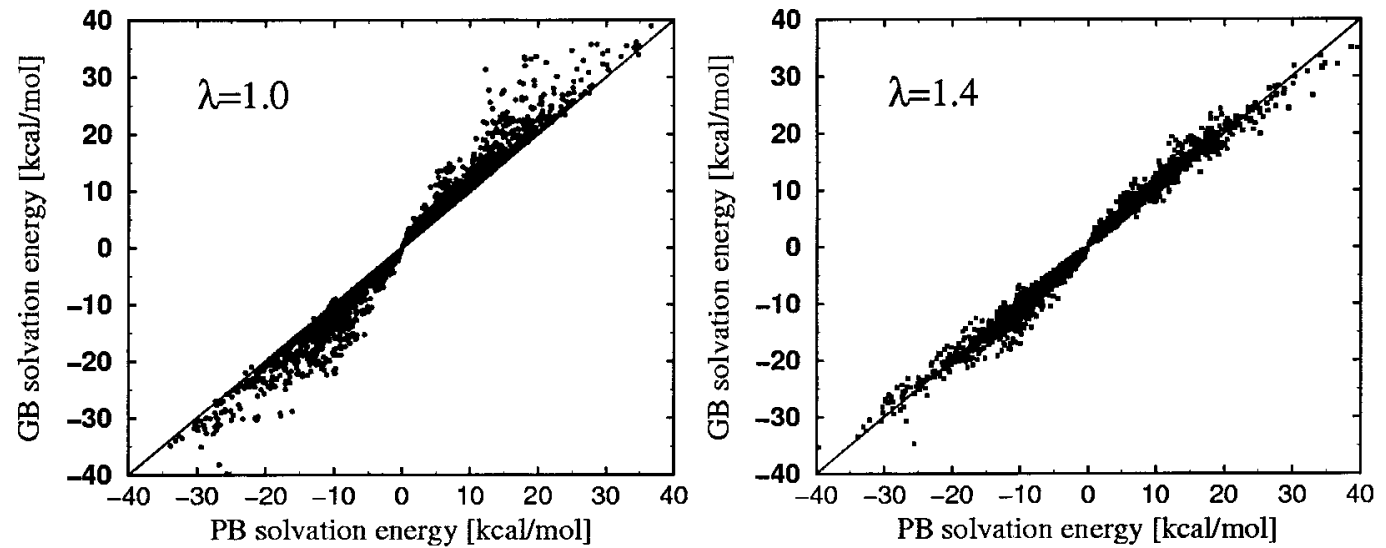

Figure 4. Comparison of the polarization part of the electrostatic charge-charge interactions $\Delta W_{i j}$ in myoglobin for GB and PB models for different values of $\lambda$, computed over a randomly selected set of $N \approx 30000$ pairs of atoms. The line $x=y$ represents a perfect match between the GB and PB theories. Compared to the original model $(\lambda=1.0)$, the modifed model with $\lambda=1.4$ produces a narrower distribution of points around the $x=y$ line, especially when the absolute value of energy is above $10 \mathrm{kcal} / \mathrm{mol}$.
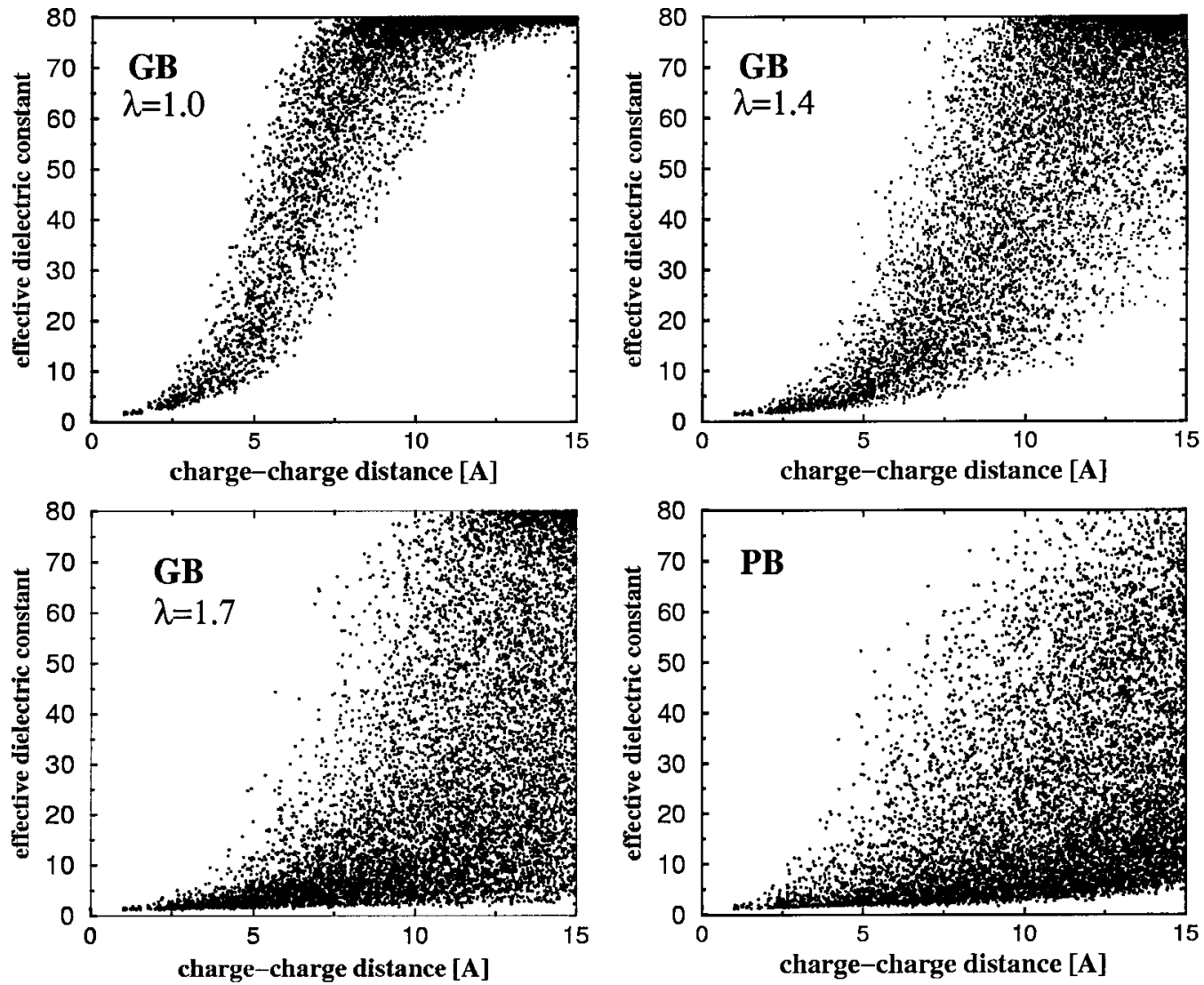

Figure 5. Effective dielectric constant $\epsilon_{i j}=W_{i j} r_{i j} /\left(q_{i} q_{j}\right)$ in myoglobin for the GB model with different values of $\lambda$, and for the PB model, computed over a randomly selected set of $N \approx 30000$ pairs of atoms.

protein is expected to present a difficult test to the GB model, as it has a number of significantly buried residues, characterized by very large $\mathrm{p} K$ shifts. We can use the protein to probe the limitations of the present theory by comparing the PB and GB predictions to each other. We have used a $1.9 \AA$ resolution X-ray structure ${ }^{45}$ of halobacterium bacteriorhodopsin (PDB Accession No. $1 \mathrm{QHJ}$ ) which has recently become available, and an older one obtained by high resolution (3.0 ̊) electron cryomicroscopy $^{47}$ (PDB Accession No. 1AT9). The all-atom charges as well as hydrogen coordinates for the retinal group of $1 \mathrm{QHJ}$ structure have been kindly provided by V. Spassov. ${ }^{46}$ We have used CHARMM charges and minimized the entire structure using the CHARMM22 force field. In the case of 1AT9 we simply use the united-atom representation of the retinal carbon atoms, keeping them uncharged. The protein charges are taken from AMBER. For both structures, Bondi radii are used. Results of the calculations of $\mathrm{p} K_{1 / 2}$ values for a set of biologically important groups in bacteriorhodopsin are shown in Table 2. All residues not shown are kept in a fixed protonation state according to their model compound protonation at $\mathrm{pH}=7.0$. No explicit water molecules are retained in either structure. We do not use explicit membranes in these calculations, and therefore do not expect the predicted $\mathrm{p} K_{1 / 2}$ values to be close to the ones obtained in the earlier work. ${ }^{31}$ The general feature of the GB model, as seen from Table 2, is an underestimation of the extreme $\mathrm{p} K$ shifts of deeply buried residues, compared to both the PB predictions and the experimental data. For the 1QHJ structure, the GB method correctly predicts an upward 
a)

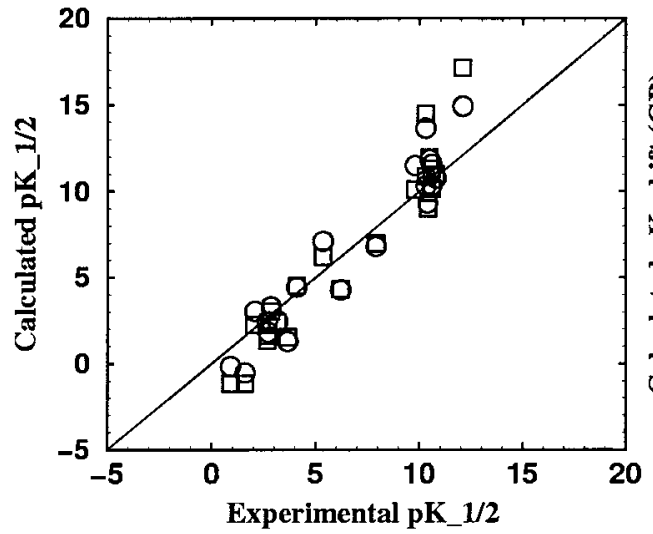

b)

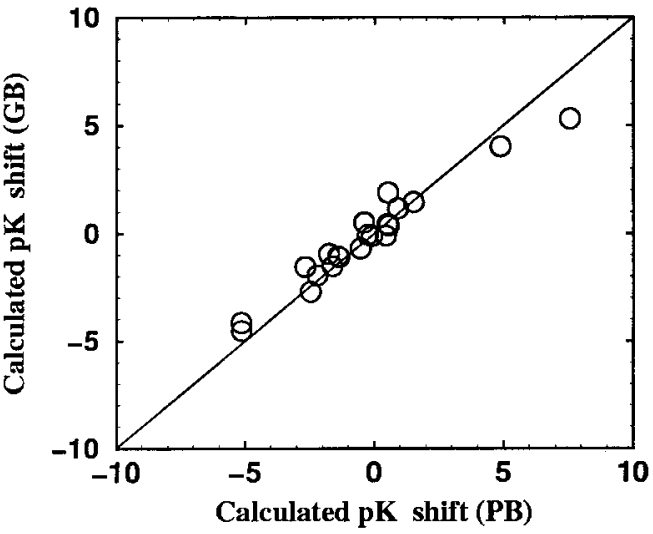

Figure 6. (a) $\mathrm{p} K_{1 / 2}$ values of 21 titratable residue in triclinic lysozyme calculated in GB (circles) and PB (squares) models plotted vs corresponding experimental results. The line $x=y$ represents a perfect match between the theory and experiment. (b) $\mathrm{p} K_{1 / 2}-\mathrm{p} K_{\text {mod }}$ calculated in the GB model is plotted vs the same quantity obtained by the PB approach. The line $x=y$ represents a perfect match between the two models.

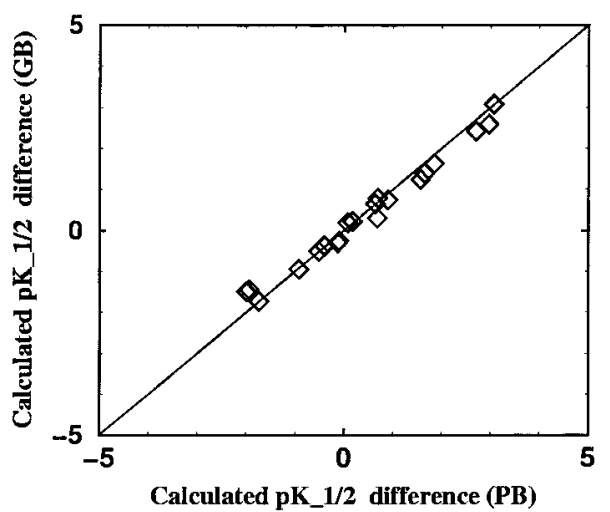

Figure 7. $\mathrm{p} K_{1 / 2}^{\mathrm{tetr}}-\mathrm{p} K_{1 / 2}^{\mathrm{tric}}$ calculated in the GB model vs the same quantity obtained by the PB approach for the set of 21 residues in the two different (triclinic and tetragonal) crystal forms of lysozyme. The line $x=y$ represents a perfect match between the two models.

TABLE 1: Values of $\mathrm{p} K_{1 / 2}$ for Myoglobin Calculated Using GB and PB Models ${ }^{a}$

\begin{tabular}{|c|c|c|c|c|c|c|}
\hline \multirow[b]{3}{*}{ site } & \multirow{3}{*}{$\begin{array}{l}\text { exptl } \\
\mathrm{p} K_{1 / 2}\end{array}$} & \multicolumn{5}{|c|}{ calcd $\mathrm{p} K_{1 / 2}$} \\
\hline & & \multicolumn{2}{|c|}{ PB } & \multicolumn{3}{|c|}{ GB } \\
\hline & & $\bar{r}=1.4 \AA$ & $r=0.0 \AA$ & $\bar{\lambda}=1.0$ & $\lambda=1.4$ & $\overline{\lambda=1.7}$ \\
\hline HIS-12 & 6.3 & 5.33 & 5.05 & 5.63 & 4.72 & 3.06 \\
\hline HIS-24 & $<4$ & -15.37 & 5.64 & 5.57 & -7.44 & -17.76 \\
\hline HIS-36 & 8.0 & 6.08 & 7.05 & 6.85 & 6.69 & 5.5 \\
\hline HIS-48 & 5.3 & 4.60 & 5.56 & 6.01 & 5.42 & 3.9 \\
\hline HIS-64 & $<4$ & -3.73 & 3.92 & 5.01 & 3.76 & -0.83 \\
\hline HIS-81 & 6.6 & 6.94 & 7.34 & 3 & 7.06 & 6.5 \\
\hline HIS-82 & $<4$ & -3.93 & 3.90 & 5.56 & 3.89 & -0.07 \\
\hline HIS-97 & 5.6 & 6.86 & 7.55 & 7.15 & 7.52 & 7.94 \\
\hline HIS-113 & 5.4 & 3.77 & 4.84 & 5.34 & 4.35 & 1.7 \\
\hline HIS-116 & 6.5 & 6.11 & 6.39 & 6.43 & 6.12 & 5.43 \\
\hline HIS-119 & 6.1 & 2.81 & 2.40 & 2.66 & 4.46 & 2.2 \\
\hline
\end{tabular}

${ }^{a}$ In the PB model different probe radii $r(\AA)$ are used to compute solvent/solute boundary. Experimental results from ref 42 .

shift in $\mathrm{p} K_{1 / 2}$ for ASP-96 and -115 (model compound $\mathrm{p} K_{1 / 2}=$ 4.0), but fails to predict the significant downward shift for ASP212 and ASP-85, which is correctly described by the PB model. The four tyrosines are predicted by the GB model to be nontitratable below $\mathrm{pH}=12$, in agreement with experiment; however the absolute numbers are lower than those obtained in the PB approach. For the 1AT9 structure, the GB approach correctly predicts the trends in $\mathrm{p} K$ shifts for all residues, although the actual values of $\mathrm{p} K_{1 / 2}$ for ASP-96 and -115 are underesti-
TABLE 2: Values of $\mathrm{p} K_{1 / 2}$ for Bacteriorhodopsin Calculated for Two Different Structures (1QHJ 1AT9) Using GB $(\lambda=$ 1.4) and PB Models ${ }^{a}$

\begin{tabular}{|c|c|c|c|c|c|}
\hline \multirow[b]{3}{*}{ site } & \multirow[b]{3}{*}{ exptl $\mathrm{p} K_{1 / 2}$} & \multicolumn{4}{|c|}{ calcd $\mathrm{p} K_{1 / 2}$} \\
\hline & & \multicolumn{2}{|c|}{$1 \mathrm{QHJ}$} & \multicolumn{2}{|c|}{ 1AT9 } \\
\hline & & PB & GB & PB & GB \\
\hline ASP-85 & $\leq 2.5$ & 3.00 & 6.85 & -4.06 & 1.22 \\
\hline ASP-96 & $\geq 9.5$ & 9.59 & 8.49 & 12.90 & 6.29 \\
\hline ASP-115 & $\geq 9.5$ & 5.68 & 7.22 & 14.21 & 7.32 \\
\hline ASP-212 & $\leq 2.5$ & -8.04 & 4.58 & 2.74 & 2.43 \\
\hline GLU-9 & N.A. & 6.35 & 8.56 & 4.736 & 4.52 \\
\hline GLU-204 & N.A. & -0.50 & 7.34 & 8.917 & 5.62 \\
\hline TYR-57 & $\geq 12$ & $>30.000$ & 24.93 & $>30.00$ & 15.50 \\
\hline TYR-79 & $\geq 12$ & 28.75 & 25.08 & 19.91 & 13.89 \\
\hline TYR-83 & $\geq 12$ & 17.16 & 16.12 & 22.89 & 15.74 \\
\hline TYR-185 & $\geq 12$ & $>30.00$ & 29.08 & 29.54 & 14.77 \\
\hline ARG-82 & N.A. & 21.13 & 15.29 & 17.00 & 12.17 \\
\hline
\end{tabular}

${ }^{a}$ Experimental results reproduced from ref 31 .

TABLE 3: Values of $p K_{1 / 2}$ for GLU Dipeptide calculated using GB and PB models

\begin{tabular}{lccc}
\hline & \multicolumn{3}{c}{ calcd $\mathrm{p} K_{1 / 2}$} \\
\cline { 2 - 4 } \multicolumn{1}{c}{ site } & & \multicolumn{2}{c}{$\mathrm{GB}$} \\
\cline { 2 - 4 } NTGLU-1 & 7.94 & 8.06 & $\lambda=1.4$ \\
GLU-1 & 4.96 & 4.62 & 8.03 \\
GLU-2 & 4.24 & 4.23 & 4.60 \\
CTGLU-2 & 2.99 & 2.94 & 2.99
\end{tabular}

mated. In general, we observe that the $\mathrm{p} K_{1 / 2}$ values predicted by the GB and the PB methods differ most when the shift from the model compound $\mathrm{p} K$ is expected to be very large. The absolute value of the shifts are larger in the PB method. We also find that, as in the case of buried residues in myoglobin, choosing a larger $\lambda$ value results in better agreement between the two approaches for these residues (results not shown). This is consistent with our earlier observation that although for proteins the size of myoglobin the $\lambda=1.4 \mathrm{~GB}$ model is closest to the PB model overall, an even higher value of $\lambda$ may be more appropriate for the deep interior regions.

3.3.4. Small Molecules. Finally, we check that setting $\lambda=$ 1.4 , as opposed to $\lambda=1$, has little effect on small molecule $\mathrm{p} K$ calculations. We use a GLU-GLU dipeptide in a standard extended conformation as a model of a small molecule.

We compare the $\mathrm{p} K_{1 / 2}$ values for the GLU-GLU dipeptide calculated within PB and GB methods for different values of 
$\lambda$, Table 3. As expected, all three calculations yield numbers that are very close to each other. Note that the same change in $\lambda$ results in a large qualitative difference in $\mathrm{p} K_{1 / 2}$ of buried residues in myoglobin, Table 1.

\section{Conclusions}

In this work the pairwise, analytic generalized Born approximation is modified to better describe large interior regions of macromolecules. The modified model is systematically applied to the computation of $\mathrm{p} K_{1 / 2}$ values for a series of residues in lysozyme, myoglobin, and bacteriorhodopsin. The predicted values show good overall agreement with experiment and analogous calculations based on numerical solution of the PoissonBoltzmann equation, with the exception of some residues in bacteriorhodopsin that exhibit very large $\mathrm{p} K$ shifts. We conclude that caution must be used when the current method is applied to deeply buried residues, especially if the expected $\mathrm{p} K$ shifts are greater than 5. It is also worth remembering that the continuum model itself may break down for some of the systems considered here.

The agreement between the two models becomes remarkable when the GB approach is used to evaluate the difference in titration behavior associated with conformational change: when applied to the calculation of the variation of $\mathrm{p} K_{1 / 2}$ values of the same residues in two different crystal forms of lysozyme, the GB model predictions are almost identical to those obtained by the PB method (correlation coefficient 0.994). This feature of the modified GB model, combined with its computational speed, makes it particularly attractive in situations when a large number of conformers need to be processed. In particular, it has recently become clear ${ }^{37,38,48}$ that multiple conformers of a protein must be taken into account for an accurate prediction of its titration properties. Here an effective strategy may involve using the relatively expensive PB method only once, in the beginning of the calculation, and then applying the fast GB model many times to evaluate the difference in electrostatic free energy between various possible conformers. We also note that many proteins, such as bacteriorhodopsin, are known to have large interior cavities, some containing water. These water molecules must be carefully treated within the general electrostatic model used here. In this respect, the GB model may be useful, as it would allow one to process in a reasonable time a large number of conformers corresponding to different orientations of the water molecules.

The modified theory gives good performance over a wide range of titratable residues in proteins, and the modifications have little effect on the established performance of the GB model on small molecules. Therefore the same parameter set can be used for a wide range of molecular shapes and sizes. The modified method may also be used in rapid calculations of electrostatic forces in molecular dynamics. In the latter case one can hope to eliminate the need for explicit solvent simulations which is particularly time-consuming for large molecules.

Acknowledgment. The authors thank D. Lidsky for the help with the $\mathrm{C}++$ implementation of the GB model, and V. Spassov for preparing the set of coordinates and charges needed for the bacteriorhodopsin calculations. The work was supported by NIH Grant GM57513.

\section{References and Notes}

(1) Cramer, C. J.; Truhlar, D. G. Chem. Rev. 1999, 99, 2161

(2) Bashford, D.; Karplus, M. Biochemistry 1990, 29, 10219.
(3) Honig, B.; Sharp, K.; Yang, A. J. Phys. Chem. 1993, 97, 1101.

(4) Bashford, D. Curr. Opin. Struct. Biol. 1991, 1, 175.

(5) Davis, M. E.; McCammon, J. A. Chem. Rev. 1993, 93, 509.

(6) Edinger, S. R.; Cortis, C.; Shenkin, P. S.; Friesner, R. A. J. Phys. Chem. B 1997, 101, 1190.

(7) Honig, B.; Nicholls, A. Science 1995, 268, 1144.

(8) Warwicker, J.; Watson, H. C. J. Mol. Biol. 1982, 157, 671.

(9) Gilson, M. K. Curr. Opin. Struct. Biol. 1995, 5, 216. 3765 .

(10) Dominy, B. N.; Brooks, C. L., III. J. Phys. Chem. B 1999, 103,

(11) Still, W. C.; Tempczak, A.; Hawley, R. C.; Hendrickson, T. J. Am. Chem. Soc. 1990, 112, 6127.

(12) Jayaram, B.; Liu, Y.; Beveridge, D. J. Chem. Phys. 1998, 109, 1465.

(13) Hawkins, G. D.; Cramer, C. J.; Truhlar, D. G. Chem. Phys. Lett. 1995, 246, 122 .

(14) Hawkins, G. D.; Cramer, C. J.; Truhlar, D. G. J. Phys. Chem. 1996, $100,19824$.

(15) Srinvasan, J.; Trevathan, M. W.; Beroza, P.; Case, D. A. Theor Chem. Acc. 1999, 101, 426.

(16) Qiu, D.; Shenkin, P.; Hollinger, F.; Still, W. J. Phys. Chem. 1997, 101,3005

(17) Luo, R.; Head, M. S.; Moult J.; Gilson, M. K. J. Am. Chem. Soc 1998, 120,6138 .

(18) Jackson, J. D. Classical Electrodynamics; John Wiley \& Sons: New York, 1975

(19) Schaefer, M.; Karplus, M. J. Phys. Chem. 1996, 100, 1578.

(20) Schaefer, M.; Froemmel, C. J. Mol. Biol. 1990, 216, 1045.

(21) Richards, F. M. Annu. Rev. Biochem. Bioeng. 1977, 6, 151.

(22) Scarsi, M.; Apostolakis, J.; Calfisch, A. J. Phys. Chem. A 1997, 101, 8098.

(23) Richards, F. M. J. Mol. Biol. 1974, 82, 1.

(24) Levitt, M.; Gerstein, M.; Huang, E.; Subbiah, S.; Tsai, J. Аnnu. Rev. Biochem. 1997, 66, 549.

(25) Hawkins, G. D.; Cramer, C. J.; Truhlar, D. G. Chem. Phys. Lett 1995, 246, 122

(26) Bashford, D. An Object-Oriented Programming Suite for Electrostatic Effects in Biological Molecules. In Lecture Notes in Computer Science; Ishikawa, Y., Oldehoeft, R. R., Reynders, J. V. W., Tholburn, M., Eds.; Springer: Berlin, 1997; p 233.

(27) Cornell, W. D.; Cieplak, P.; Bayly, C. I.; Gould, I. R.; Merz, K. M., Jr.; Ferguson, D. M.; Spellmeyer, D. C.; Fox, T.; Caldwell, J. W.; Kollman P. A. J. Am. Chem. Soc. 1995, 117, 5179.

(28) Bondi, A. J. Chem. Phys. 1964, 64, 441.

(29) Beroza, P.; Case, D. A. Methods Enzymol. 1998, 295, 170.

(30) Bashford, D.; Case, D. A.; Dalvit, C.; Tennant, L.; Wright, P. E. Biochemistry 1993, 32, 8045.

(31) Bashford, D.; Gerwert, K. J. Mol. Biol. 1992, 224, 473.

(32) Bashford, D.; Karplus, M. J. Phys. Chem. 1991, 95, 9556.

(33) Beroza, P.; Fredkin, D. R.; Okumara, M. Y.; Feher, G. Proc. Natl. Acad. Sci. U.S.A. 1991, 88, 5804.

(34) Kuriyan, J.; Wiltz, S.; Karplus, M.; Petsko, J. J. Mol. Biol. 1986, 192, 133.

(35) Bentley, G.; Duee, E.; Mason, S.; Nunes, A. J. Chem. Phys. 1979, 76,817 .

(36) The PB results presented here differ from those of ref 2 because a different coordinate set is used.

(37) You, T.; Bashford, D. Biophys. J. 1995, 69, 1721.

(38) Alexov, E. G.; Gunner, M. R. Biochemistry 1999, 38, 8253.

(39) Beroza, P.; Case, D. A. J. Phys. Chem. 1996, 100, 20156.

(40) Bosch, R.; Lautenschlager, P.; Potthast, L.; Stapelmann, J. J. Cryst. Growth 1992, 122, 310.

(41) Computations performed on a HP-735 machine.

(42) Cocco, M.; Kao, Y.; Phillips, A.; Lecomte, J. Biochemistry 1992, 31,6481 .

(43) Sampogna, R.; Honig, B. Biophys. J. 1994, 66, 1341.

(44) Scharnagl, C.; Hettenkofer, J.; Fischer, S. F. J. Phys. Chem. 1995, $99,7787$.

(45) Belrhali, H.; Nollert, P.; Royant, A.; Menzel, C.; Rosenbusch, J. P.; Landau, E. M.; Pebay-Peyroula, E. Structure 1999, 7, 909.

(46) Spassov, V. Private communications. A density functional theory was used to compute the charges.

(47) Henderson, R.; Bawldwin, J. M.; Ceska, T. A.; Zemlin, F.; Beckmann, E.; Downing, K. H. J. Mol. Biol. 1990, 213, 899.

(48) Zhou, Huan-Xiang.; Vijayakumar, M. J. Mol. Biol. 1997, 267, 1002. 